\title{
SANS and DLS Studies of Protein Unfolding in Presence of Urea and Surfactant
}

\author{
V. K. Aswal ${ }^{1}$, S. N. Chodankar ${ }^{1}$, J. Kohlbrecher ${ }^{2}$, R. Vavrin $^{2}$ and A. G. Wagh $^{1}$ \\ ${ }^{1}$ Solid State Physics Division, Bhabha Atomic Research Centre, Mumbai 400085, India \\ ${ }^{2}$ Laboratory for Neutron Scattering, ETH Zurich \& Paul Scherrer Institut, CH-5232 Villigen PSI, Switzerland
}

\begin{abstract}
Small-angle neutron scattering (SANS) and dynamic light scattering (DLS) have been used to study conformational changes in protein bovine serum albumin (BSA) during its unfolding in presence of protein denaturating agents urea and surfactant. On addition of urea, the BSA protein unfolds for urea concentrations greater than $4 \mathrm{M}$ and acquires a random coil configuration with its radius of gyration increasing with urea concentration. The addition of surfactant unfolds the protein by the formation of micelle-like aggregates of surfactants along the unfolded polypeptide chains of the protein. The fractal dimension of such a protein-surfactant complex decreases and the overall size of the complex increases on increasing the surfactant concentration. The conformation of the unfolded protein in the complex has been determined directly using contrast variation SANS measurements by contrast matching the surfactant to the medium. Results of DLS measurements are found to be in good agreement with those obtained using SANS.
\end{abstract}

Keywords: Protein unfolding, small-angle neutron scattering, dynamic light scattering

PACS: $61.12 . E x$, 87.14.Ee, 87.15.Nn

\section{INTRODUCTION}

The function of a protein depends absolutely on its three-dimensional folded structure. Protein unfolding process involves the disruption of H-bonds, disulphide bonds, salt bridges and hydrophobic interactions, leading to its successive alteration of quaternary, tertiary, and secondary structure. However, peptide bonds are not broken leaving the primary structure unaltered. Protein unfolding is one of the most widely studied topics in molecular biology due to its wide spread application in the industrial and scientific world [1]. The unfolding process can be brought about by various means and conditions. Each different route of unfolding has its own application and advantage in material processing and basic sciences. Urea is being used for long time to understand the fundamental process of protein folding/unfolding. On the other hand, the surfactant induced unfolding is known to play an important role in the pharmaceutical and cosmetic industry. Urea is known for its water breaking ability, causing an increase in the solubility of hydrophobic groups, thereby being able to solubilize both the hydrophobic and hydrophilic patches of the protein macromolecule leading to the unfolding of the whole protein. Protein unfolding in the case of amphiphilic molecules such as surfactant is caused due to binding of these molecules to the hydrophobic patches of the protein.

Earlier studies have proposed that the addition of urea unfolds a protein into a polypeptide chain which acquires a random coil conformation [2]. In the presence of surfactant, the binding of surfactant on protein results in micelle-like aggregates enclosing the hydrophobic patches on the protein backbone. This leads to acquisition of a necklace-bead structure of the protein surfactant complex [3]. Herein, we show the results of small-angle neutron scattering (SANS) and dynamic light scattering (DLS) to probe conformational changes during the protein unfolding induced by on addition of urea and surfactant. The experiments are performed on bovine serum albumin (BSA) protein, which is one of the commonly used proteins. Protein unfolding in presence of urea is studied over a wide concentration range of urea $(0$ $8 \mathrm{M})$. The systematic structural changes during protein unfolding on addition of varying concentration of anionic surfactant sodium dodecyl sulfate (SDS) have been examined. SANS with the possibility to vary the contrast is an ideal technique for studying hydrogenous systems such as protein solution. This technique provides information about the geometry and conformation of the scattering particles [4]. DLS is a complimentary technique to SANS, which gives size information by measuring the diffusion coefficient of the particle [5]. 


\section{EXPERIMENTAL}

BSA protein (catalogue no. 05480), SDS surfactant (catalogue no. 71727) and urea were purchased from Fluka. Samples for SANS experiments were prepared by dissolving known amount of BSA and other additives (surfactant or urea) in a buffer solution of $\mathrm{D}_{2} \mathrm{O}$. The use of $\mathrm{D}_{2} \mathrm{O}$ as solvent instead of $\mathrm{H}_{2} \mathrm{O}$ provides better contrast for hydrogenous components in neutron experiments. The interparticle interactions in these systems were minimized by preparing the samples in acetate buffer solution at $\mathrm{pH} 5.4$, which is close to the isoelectric pH of BSA (4.9), and at high ionic strength of $0.5 \mathrm{M} \mathrm{NaCl}$. Small-angle neutron scattering experiments were performed on the SANS-I instrument at the Swiss Spallation Neutron Source, SINQ, Paul Scherrer Institut, Switzerland [6]. The mean wavelength of the incident neutron beam was 6 $\AA$ with a wavelength resolution of approximately $10 \%$. The scattered neutrons were detected using twodimensional $96 \mathrm{~cm} \times 96 \mathrm{~cm}$ detector. The experiments were performed at two sample-to-detector distances of 2 and $8 \mathrm{~m}$, respectively to cover the data in the wave vector transfer $Q$ range of 0.007 to $0.25 \AA^{-1}$. The measured SANS data were corrected and normalized to a cross-sectional unit using BerSANS-PC data processing software [7]. DLS measurements on above samples were carried out using a commercial ALV/LSE-5003 light scattering instrument featuring a multiple tau digital correlator. The light source was a helium neon laser operated at $6328 \AA$ and the measurements were performed at a scattering angle of $90^{\circ}$. The temperature was kept fixed at $30^{\circ} \mathrm{C}$ for all the measurements.

\section{DATA ANALYSIS}

\section{Small-angle neutron scattering}

In small-angle neutron scattering one measures the coherent differential scattering cross-section per unit volume $[d \Sigma / d \Omega(Q)]$ as a function of $Q$. For a system of monodispersed interacting protein macromolecules, $d \Sigma / d \Omega(Q)$ can be expressed as [8].

$$
\frac{d \Sigma}{d \Omega}(Q)=N_{p} V_{p}^{2}\left(\rho_{p}-\rho_{s}\right)^{2}\left[\left\langle F(Q)^{2}\right\rangle+\langle F(Q)\rangle^{2}\left(S_{p}(Q)-1\right)\right]+B
$$

where $N_{p}$ is the number density and $V_{p}$ is the volume of the protein macromolecule. $\rho_{p}$ and $\rho_{s}$ are the scattering length density of the protein and the solvent, respectively. $F(Q)$ is the single particle form factor and $S_{p}(Q)$ is the interparticle structure factor. In general, charged colloidal systems such as protein solutions show a correlation peak in the SANS distribution [9]. The peak arises because of the interparticle structure factor $S_{p}(Q)$ and indicates the presence of electrostatic interaction between the colloids. In the case of a solution with low protein concentration, having high salt concentration and $\mathrm{pH}$ close to isoelectric point of the protein, $S_{p}(Q)$ can be approximated to unity as the interparticle interactions are minimized so that

$\frac{d \Sigma_{n}}{d \Omega}(Q)=N_{p} V_{p}^{2}\left(\rho_{p}-\rho_{s}\right)^{2}\left\langle F(Q)^{2}\right\rangle+B$

$B$ is a constant term that represents the incoherent scattering background, which is mainly due to hydrogen in the sample. The single particle form factor of the protein macromolecules in their native conformation has been calculated by treating them as prolate ellipsoids. For such an ellipsoidal particle

$$
\begin{aligned}
& \left\langle F^{2}(Q)\right\rangle=\int_{0}^{1}\left[F(Q, \mu)^{2} d \mu\right] \\
& F(Q, \mu)=\frac{3(\sin x-x \cos x)}{x^{3}} \\
& x=Q\left[a^{2} \mu^{2}+b^{2}\left(1-\mu^{2}\right)\right]^{1 / 2}
\end{aligned}
$$

where $a$ and $b$ are, respectively, the semimajor and semiminor axes of the ellipsoidal protein macromolecules and $\mu$ is the cosine of the angle between the directions of $a$ and the wave vector transfer $Q$.

In order to describe the unfolding of protein in presence of urea is modeled using a random coil Gaussian conformation

$\frac{d \Sigma_{g}}{d \Omega}(Q)=I_{0}\left[Q^{2} R_{g}^{2}-1+\exp \left(-Q^{2} R_{g}^{2}\right)\right] /\left(Q R_{g}\right)^{4}$

where $R_{g}$ is the radius of gyration of the unfolded protein polypeptide chain.

The unfolding of protein in presence of surfactant has been treated using the necklace model of proteinsurfactant complexes that assumes micelle-like clusters of surfactant randomly distributed along the unfolded polypeptide chain. The cross-section for such a system can be written as [10]

$\frac{d \Sigma_{s}}{d \Omega}(Q)=\frac{N_{1}^{2}}{N_{p} N}\left(b_{m}-V_{m} \rho_{s}\right)^{2} P_{m}(Q) S_{f}(Q)+B$

where $N_{l}$ is the number density of the total surfactant moecules in solution. $V_{m}$ the volume of the micelle and $N$ the number of such micelles attached to a polypeptide chain. $b_{m}$ represents the scattering length of the surfactant molecule. $P_{m}(Q)$ denotes the normalized intraparticle structure factor of a single micelle-like cluster. $S_{f}(Q)$ for such a system is expressed as 
$S_{f}(Q)=1+\frac{1}{(Q R)^{D}} \frac{D \Gamma(D-1)}{\left[1+(Q \xi)^{-2}\right]^{[(D-1) / 2]}} \times \sin \left[(D-1) \tan ^{-1}(Q \xi)\right]$

where $D$ is the fractal dimension of the micellar distribution in space and $\xi$ the correlation length that is a measure of the extent of unfolding of the polypeptide chain.

\section{Dynamic light scattering}

The signal generated by the light scattering from diffusing particles can be analyzed by its intensity autocorrelation function $G^{I}(\tau)$ [5]

$$
G^{I}(\tau)=\langle I(t) I(t+\tau)\rangle
$$

where $I(t)$ is the scattered light intensity at time $t$ and $I(t+\tau)$ the scattered light intensity at time $t$ plus a log time $\tau$. The normalized intensity autocorrelation function $g^{I}(\tau)$ is

$g^{I}(\tau)=\frac{G^{I}(\tau)}{\langle I(t)\rangle^{2}}$

The electric field autocorrelation function $g^{E}(\tau)$ is related to the normalized intensity autocorrelation function by the Siegert relation

$$
g^{I}(\tau)=1+C\left[g^{E}(\tau)\right]^{2}
$$

where $C$ is an experimental parameter which mainly depends on the detection optics and alignment. For a mono-disperse system of particles, $g^{E}(\tau)$ follows a simple exponential decay with decay constant $\gamma$.

$$
g^{E}(\tau)=\exp [-\gamma \tau]
$$

The average decay rate $(\gamma)$ of $g^{E}(\tau)$ has been estimated using a monomodal fit. The apparent diffusion coefficient $\left(D_{a}\right)$ is obtained from the relation $\gamma=D_{a} Q^{2}$ and the corresponding effective hydrodynamic size $\left(R_{H}\right)$ calculated using StokesEinstein relationship as given by

$R_{H}=\frac{k_{B} T}{6 \pi \eta D_{a}}$

where $k_{B}$ is the Boltzmann constant, $T$ is the temperature and $\eta$ is the viscosity of the solvent.

\section{RESULTS AND DISCUSSION}

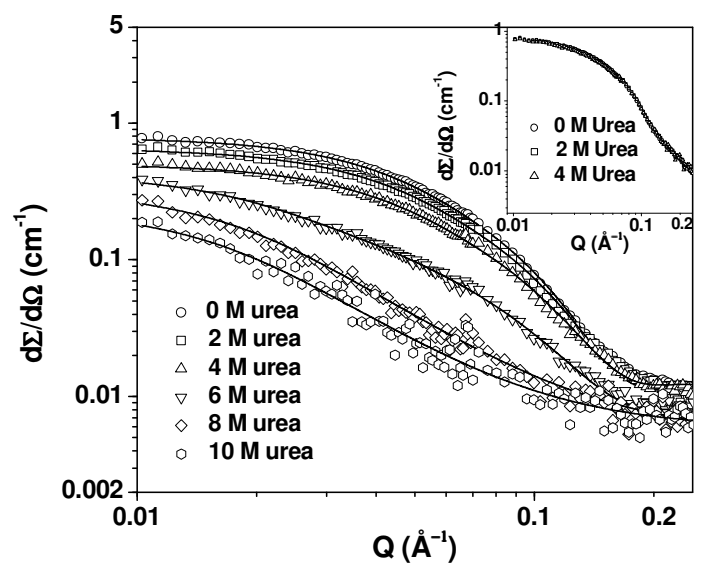

FIGURE 1. SANS data for $1 \mathrm{wt} \% \mathrm{BSA}$ in presence of varying concentration of urea. Inset shows the scaling of data up to urea concentration of $4 \mathrm{M}$.

TABLE 1. Fitted parameters of SANS analysis for $1 \mathrm{wt} \%$ BSA in presence of varying urea concentrations. The protein has a prolate ellipsoidal shape up to $4 \mathrm{M}$ urea beyond this concentration it unfolds into a random coil conformation.

\begin{tabular}{|c|c|c|c|}
\hline $\begin{array}{c}\text { [Urea] } \\
(\mathrm{M})\end{array}$ & $\begin{array}{c}\text { Semi-minor } \\
\text { axis } \\
a(\AA)\end{array}$ & $\begin{array}{c}\text { Semi-major } \\
\text { axis } \\
b=c(\AA)\end{array}$ & $R_{g}(\AA)$ \\
\hline 0 & $22.2 \pm 0.8$ & $71.0 \pm 5.1$ & - \\
\hline 2 & $22.2 \pm 0.8$ & $71.0 \pm 5.1$ & - \\
\hline 4 & $22.2 \pm 0.8$ & $71.0 \pm 5.1$ & - \\
\hline 6 & - & - & $55.0 \pm 2.9$ \\
\hline 8 & - & - & $84.0 \pm 4.1$ \\
\hline 10 & - & - & $93.5 \pm 6.4$ \\
\hline
\end{tabular}

SANS data for $1 \mathrm{wt} \% \mathrm{BSA}$ in presence of a varying concentration of urea are shown in figure 1 . The scattering cross-section decreases with increasing urea concentration. It is observed that up to $4 \mathrm{M}$ concentration of urea, there is a continuous decrease in the scattering cross-section, however the functionality of the scattering pattern does not change. The inset of the figure 1 shows the scaling of the data suggesting the same functionality of the scattering profiles for urea concentrations in the range 0 to $4 \mathrm{M}$. The decrease in scattering cross-section can be explained in terms of decrease in contrast $\left(\rho_{p}-\rho_{s}\right)^{2}$ as the scattering length density of deuterated solvent $\left(\rho_{s}\right)$ decreases on addition of hydrogenous urea to protein solution [11]. There is a change in the functionality of the scattering profile beyond $4 \mathrm{M}$ urea and it is interpreted in terms of unfolding of the protein. It is believed that the solvation of hydrophobic portions of the protein at 
high urea concentrations leads to the unfolding of a protein. The unfolded protein is fitted as random Gaussian coil using equation (6). It is found that radius of gyration $\left(R_{g}\right)$ of the unfolded protein increases with increasing urea concentration. The value of $R_{g}$ increases from 55 to $93.5 \AA$ as the urea concentration is increased from $6 \mathrm{M}$ to $10 \mathrm{M}$ (Table 1).

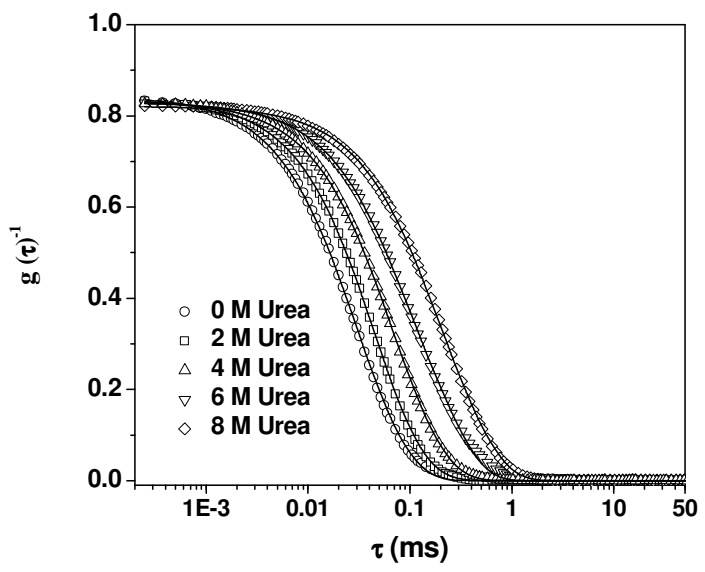

FIGURE 2. DLS data for $1 \mathrm{wt} \% \mathrm{BSA}$ in presence of varying concentration of urea.

TABLE 2. Fitted parameters of DLS analysis for $1 \mathrm{wt} \%$ BSA in presence of varying urea concentrations.

\begin{tabular}{|c|c|c|c|}
\hline $\begin{array}{c}\text { [Urea] } \\
(\mathrm{M})\end{array}$ & $\begin{array}{c}\text { Diffusion } \\
\text { coefficient } \\
D_{a}\left(10^{-8} \mathrm{~cm}^{2} / \mathrm{s}\right)\end{array}$ & $\begin{array}{c}\text { Solvent } \\
\text { viscosity } \\
\eta(\mathrm{mPa} \mathrm{s})\end{array}$ & $\begin{array}{c}\text { Hydrodynamic } \\
\text { radius } \\
R_{H}(\AA)\end{array}$ \\
\hline 0 & 64.3 & 1.03 & 33.5 \\
\hline 2 & 63.6 & 1.12 & 34.1 \\
\hline 4 & 61.2 & 1.25 & 34.5 \\
\hline 6 & 43.6 & 1.44 & 68.5 \\
\hline 8 & 18.3 & 1.71 & 136.5 \\
\hline
\end{tabular}

DLS data on addition of urea to protein solution are shown in Figure 2. DLS measures the time-dependent fluctuations in the intensity of scattered light [5]. These fluctuations happen as a result of the Brownian motion. Small particles diffuse rapidly and yield fast fluctuations, whereas large particles and aggregates generate relatively slow fluctuations. The rate of the fluctuations is determined through the autocorrelation analysis technique. The calculated autocorrelation function enables the determination of the diffusion coefficient [Equation (11)], which then can be converted to a size using the Stokes-Einstein relationship [Equation (13)]. There is a decrease in the average decay constant indicating a slowing down of the diffusion of the protein macromolecules on increasing urea concentration. The calculated hydrodynamic size from the DLS data is shown in Table 2. The hydrodynamic size remains similar up to $4 \mathrm{M}$ urea concentration and increases beyond this concentration as the protein unfolds, which is consistent with the SANS results (Table 1).

Figure 3 shows SANS data for $1 \mathrm{wt} \%$ BSA in presence of varying SDS concentration. Based on the features of the scattering profiles, the data can be grouped in two different sets as the surfactant concentration is increased. The first data set corresponds to proteins at low surfactant concentrations ( 0 to $10 \mathrm{mM})$, where the scattering data show similar behavior to that of pure protein solution. In this data set, the overall scattering cross-section increases with increase in surfactant concentration. It can be explained in terms of Equation (1) if the individual surfactant molecules bind to protein and the volume of the scattering particle increases. The features of the scattering data in the second data set at higher surfactant concentrations $(>10 \mathrm{mM})$ are very different to those of the first data set. One of the interesting features is the linearity of the scattering profiles on log-log scale in the intermediate $Q$ range with a $Q$ range of linearity increasing with surfactant concentration. This is an indication of formation of fractal structure by the protein-surfactant complex [12]. The build up of scattering cross-section in the higher cut-off of the linearity of scattering data suggests the formation of surfactant aggregates and the lower cutoff corresponds to the overall size of the proteinsurfactant complex. It is observed that the position of high $Q$ cut-off remains almost same while the position of low $Q$ cut-off shifts to smaller $Q$ values with increasing surfactant concentration. The calculated structural parameters in this system are given in Table 3.

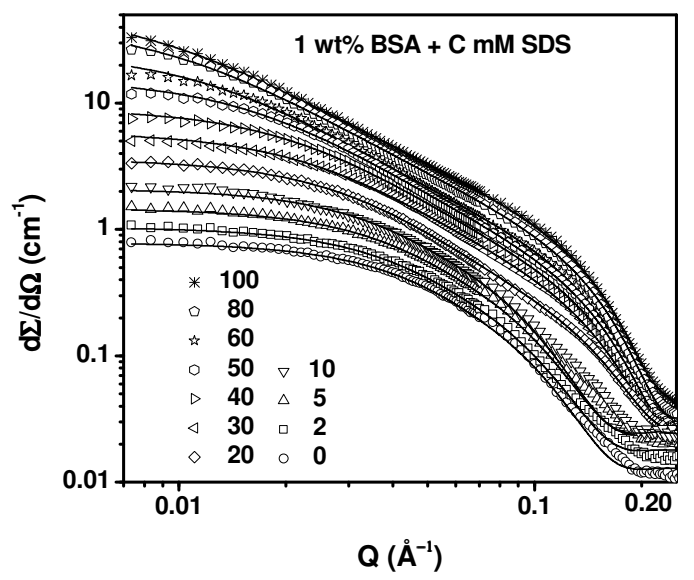

FIGURE 3. SANS data for $1 \mathrm{wt} \% \mathrm{BSA}$ in presence of varying concentration of surfactant.

At low surfactant concentrations, Table 3 shows changes in the dimensions of the protein on increasing binding of surfactant molecules as a function of surfactant concentration. The semiminor axis remains 
almost same while the semimajor axis increases with increasing surfactant concentration. The size of the protein elongates from 70 to $88 \AA$ along the semimajor axis on the addition of $10 \mathrm{mM}$ of surfactant. Similar results of the elongation of the BSA protein have also been observed with cationic surfactant Azobenzene trimethyl ammonium bromide. It is believed that the six protein sub-domains forming BSA remain intact but separates from each other, leading to an elongation of the protein on addition of surfactant [13]. The fractal structure of the protein-surfactant complex at higher surfactant concentrations is modeled on the basis of the necklace model which considers micellelike clusters of the surfactant formed along the unfolded polypeptide chain of the protein. It is found that the fractal dimension decreases and the overall size of the complex increases on increasing surfactant concentration. The size of micelle-like clusters $(R)$ does not change while the number of such micelle-like clusters $(N)$ in protein-surfactant complex increases with the surfactant concentration (Table 3).

TABLE 3. Fitted parameters of SANS analysis of proteinsurfactant complex for

(a) $1 \mathrm{wt} \%$ BSA with low SDS concentrations 0 to $10 \mathrm{mM}$

\begin{tabular}{|c|c|c|}
\hline $\begin{array}{c}{[\mathrm{SDS}]} \\
(\mathrm{mM})\end{array}$ & $\begin{array}{c}\text { Semi-major } \\
\text { axis } \\
a(\AA)\end{array}$ & $\begin{array}{c}\text { Semi-minor } \\
\text { axis } \\
b=c(\AA)\end{array}$ \\
\hline 0 & $70.2 \pm 5.1$ & $22.2 \pm 0.8$ \\
\hline 2 & $77.3 \pm 5.8$ & $22.2 \pm 0.8$ \\
\hline 5 & $80.0 \pm 6.1$ & $22.2 \pm 0.8$ \\
\hline 10 & $88.0 \pm 6.4$ & $23.0 \pm 0.9$ \\
\hline
\end{tabular}

(b) $1 \mathrm{wt} \%$ BSA with high SDS concentrations 20 to $100 \mathrm{mM}$

\begin{tabular}{|c|c|c|c|c|}
\hline $\begin{array}{c}{[\mathrm{SDS}]} \\
(\mathrm{mM})\end{array}$ & $\begin{array}{c}\text { Fractal } \\
\text { dimension } \\
D\end{array}$ & $\begin{array}{c}\text { Correlation } \\
\text { length } \\
\xi(\AA)\end{array}$ & $\begin{array}{c}\text { Micelle } \\
\text { radius } \\
R(\AA)\end{array}$ & $\begin{array}{c}\text { No. of } \\
\text { micelles } \\
N\end{array}$ \\
\hline 20 & $2.27 \pm 0.15$ & $38.0 \pm 1.9$ & 18.0 & 2 \\
\hline 30 & $2.15 \pm 0.14$ & $43.0 \pm 2.7$ & 18.0 & 3 \\
\hline 40 & $2.05 \pm 0.13$ & $54.0 \pm 3.8$ & 18.0 & 4 \\
\hline 50 & $1.95 \pm 0.10$ & $67.8 \pm 4.9$ & 18.0 & 6 \\
\hline 60 & $1.88 \pm 0.09$ & $87.9 \pm 5.4$ & 18.0 & 8 \\
\hline 80 & $1.79 \pm 0.06$ & $117.9 \pm 6.9$ & 18.0 & 10 \\
\hline 100 & $1.71 \pm 0.04$ & $144.3 \pm 7.5$ & 18.0 & 13 \\
\hline
\end{tabular}

The calculated aggregation number of micelle-like clusters in the complex decreases from 51 to 42 on increasing surfactant concentration from 20 to $100 \mathrm{mM}$. It is interesting to note that these values of aggregation numbers are much smaller than as one would have found about 70 in pure surfactant solution for the similar size of micelles [14]. This indicates the participation of the hydrophobic portions of the unfolded protein chain in the micellar formation. The participation of the unfolded protein in the formation of micelle-like clusters is enhanced with the increase in unfolding and this result in decreasing aggregation number of micelle-like clusters. Also all the surfactant molecules probably participate in the micelle-like clusters to avoid the exposure of hydrophobic portions of the protein on its unfolding with increase in surfactant concentration.

The conformational changes of protein in proteinsurfactant complex have been examined by contrast variation SANS by contrast matching the surfactant. The surfactant is contrast matched using deuterated SDS (d-SDS) and the sample prepared in $\mathrm{D}_{2} \mathrm{O}$. Figure 4 shows the SANS data for $1 \mathrm{wt} \% \mathrm{BSA}$ in presence of varying d-SDS concentration. Unlike in Figure 3 where the scattering cross-section increases on increasing SDS concentration, Figure 4 shows decrease in scattering cross-section with increase in dSDS concentration. This can be understood in terms of equation (1) that the contrast of protein decreases as the size of complex increases on addition of surfactant (Tables 3).

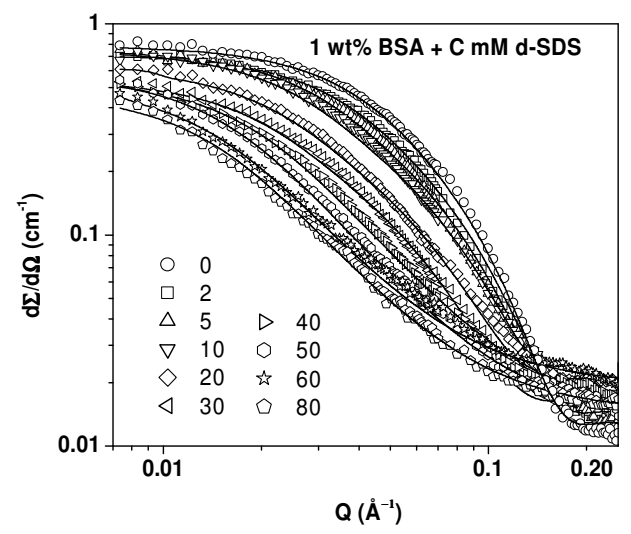

FIGURE 4. SANS data for $1 \mathrm{wt} \% \mathrm{BSA}$ in presence of varying concentration of deuterated surfactant.

Similar to Figure 3, Figure 4 also shows two sets of data as obtained the first set at low and the second set at high surfactant concentrations. The first data set corresponds to the binding of individual surfactant molecules and hence the size of the complex increases as more and more surfactant is added (Table 4). This data set is fitted with Equation (1). The analysis of the data (Table 4) gives similar dimensions of the complex as obtained in Table 3. For the data of second set micelle-like clusters of surfactant molecules are formed in the complex (Table 3). The unfolded protein in the complex is fitted by the scattering function obeying random coil Gaussian conformation. It may be mentioned that at d-SDS concentration of 20 and $30 \mathrm{mM}$, where the complex already consists of micellelike clusters the data are still best fitted with a prolate 
ellipsoidal shape of the protein. Unfolding in these systems is limited due to formation of small number of micelles in the complex (Table 3).

TABLE 4. Fitted parameters of SANS analysis of proteinsurfactant complex for $1 \mathrm{wt} \%$ BSA with varying d-SDS concentration.

\begin{tabular}{|c|c|c|c|}
\hline $\begin{array}{c}{[\mathrm{d}-\mathrm{SDS}]} \\
(\mathrm{mM})\end{array}$ & \multicolumn{2}{|c|}{$\begin{array}{c}\text { Folded } \\
\text { structure }\end{array}$} & $\begin{array}{c}\text { Unfolded } \\
\text { Structure }\end{array}$ \\
\hline $\begin{array}{c}\text { Semi-major } \\
a(\AA)\end{array}$ & $\begin{array}{c}\text { Semi-minor } \\
\text { axis } \\
b=c(\AA)\end{array}$ & $\begin{array}{c}\text { Radius of } \\
\text { gyration } \\
R_{g}(\AA)\end{array}$ \\
\hline 0 & $71.0 \pm 5.1$ & $22.2 \pm 0.8$ & - \\
\hline 2 & $77.3 \pm 5.8$ & $22.2 \pm 0.8$ & - \\
\hline 5 & $82.0 \pm 6.3$ & $22.2 \pm 0.8$ & - \\
\hline 10 & $88.0 \pm 6.4$ & $23.0 \pm 0.9$ & - \\
\hline 20 & $94.0 \pm 6.7$ & $25.8 \pm 1.1$ & - \\
\hline 30 & $99.0 \pm 7.1$ & $27.1 \pm 1.3$ & - \\
\hline 40 & - & - & $60.1 \pm 1.6$ \\
\hline 50 & - & - & $70.3 \pm 1.8$ \\
\hline 60 & - & - & $85.5 \pm 2.4$ \\
\hline 80 & - & - & $102.3 \pm 4.6$ \\
\hline
\end{tabular}

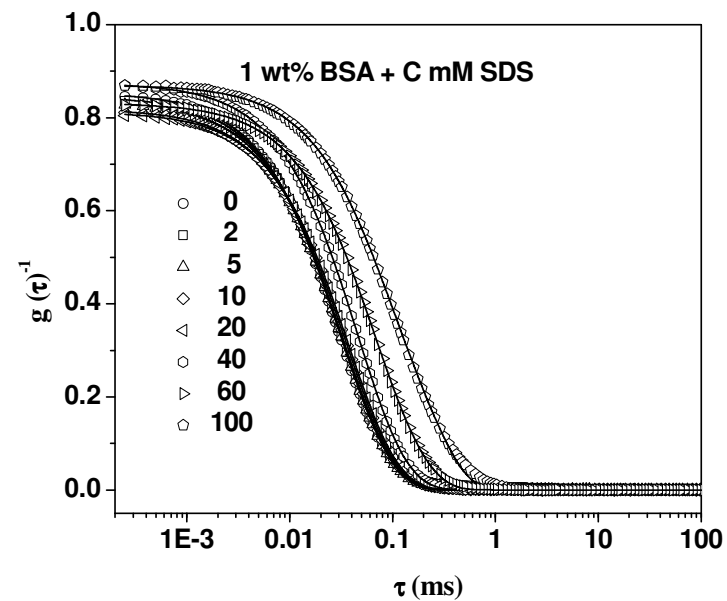

FIGURE 5. DLS data for $1 \mathrm{wt} \% \mathrm{BSA}$ in presence of varying concentration of surfactant.

DLS data for $1 \mathrm{wt} \%$ BSA on addition of surfactant are shown in Figure 5. These data show the slowing down of intensity auto correlation function with increasing surfactant concentration. The analysis suggests that irrespective of the surfactant concentration, all the data fit to the single diffusion coefficient for the structure of the protein-surfactant complex. The fitted values of the apparent diffusion coefficient $\left(D_{a}\right)$ and the corresponding hydrodynamic radius $\left(R_{h}\right)$ are given in table $\mathrm{V}$. There is decrease in the value of $D_{a}$ and increase in $R_{h}$ with increase in surfactant concentration. The diffusion coefficient decreases as the amount of surfactant in the complex increases either through the binding of surfactant molecules as individuals or through the formation of micelle-like clusters with the protein. The increase in overall size of complex is significant at higher surfactant concentrations as related to the unfolding of protein. These observations are in agreement with the results obtained using SANS. The larger values of the sizes of the complex obtained using DLS than SANS (Table 5) are expected because DLS measures structure along with its hydration.

TABLE 5. Fitted parameters as obtained by DLS for the protein-surfactant complex with $1 \mathrm{wt} \%$ BSA and varying SDS concentrations. SANS results are compared with effective size of protein-surfactant complex calculated from table III as $\left(a b^{2}\right)^{1 / 3}$ for the folded structure and as $\xi$ for the unfolded structure.

\begin{tabular}{|c|c|c|c|}
\hline $\begin{array}{c}{[\mathrm{SDS}]} \\
(\mathrm{mM})\end{array}$ & $\begin{array}{c}\text { Diffusion } \\
\text { coefficient } \\
D_{a}\left(10^{-8} \mathrm{~cm}^{2 /} \mathrm{sec}\right)\end{array}$ & $\begin{array}{c}\text { Hydrodynamic } \\
\text { radius } \\
R_{h}(\AA)\end{array}$ & $\begin{array}{c}\text { Effective } \\
\text { size } \\
R_{e}(\AA)\end{array}$ \\
\hline 0 & 64.3 & 33.5 & 32.6 \\
\hline 2 & 64.3 & 33.5 & 33.5 \\
\hline 5 & 59.0 & 36.5 & 34.1 \\
\hline 10 & 55.2 & 39.0 & 36.0 \\
\hline 20 & 51.9 & 41.5 & 38.0 \\
\hline 40 & 35.7 & 60.3 & 54.0 \\
\hline 60 & 23.6 & 91.2 & 87.9 \\
\hline 100 & 14.3 & 150.5 & 144.3 \\
\hline
\end{tabular}

\section{REFERENCES}

1. C.M. Dobson, Nature 426, 884 (2003).

2. R.S. Tu and V. Breedveld, Phys. Rev. E72, 041914 (2005).

3. S.H. Chen and J. Teixeira, Phys. Rev. Lett. 57, 2583 (1986).

4. D.I. Svergun and M.H.J. Koch, Rep. Prog. Phys. 66, 1735 (2002).

5. R. Pecora, Dynamic Light Scattering, New York, Plenum, 1985.

6. J. Kohlbrecher and W. Wagner, J. Appl. Cryst. 33, 804 (2000).

7. U. Keiderling, Appl. Phys. A 74, S1455 (2002).

8. J.S. Pedersen, Adv. Colloid Interface Sci. 70, 171 (1997).

9. S. Chodankar and V.K. Aswal, Phys. Rev. E72, 041931 (2005).

10. X.H. Guo, N.M. Zhao, S.H. Chen and J. Teixeira, Biopolymers 29, 335 (1990).

11. V.K. Aswal, J. Kohlbrecher, P.S. Goyal, H. Amenitsch and S. Bernstorff, J. Phys.: Condens. Matter 18, 11399 (2006).

12. S. Chodankar, V.K. Aswal, P.A. Hassan and A.G. Wagh, Phys. B398, 112 (2007).

13. T.C. Lee, K.A. Smith, T.A. Hatton, Biochemistry 44, 524 (2005).

14. V.K. Aswal and P.S. Goyal, Phys. Rev. E67, 051401 (2003). 